University of Wollongong

Research Online

Faculty of Engineering and Information

Faculty of Engineering and Information

Sciences - Papers: Part A

Sciences

2016

Development of a novel magnetophoresis-assisted hydrophoresis microdevice for rapid particle ordering

Sheng Yan

University of Wollongong, sy034@uowmail.edu.au

Jun Zhang

University of Wollongong, jz218@uowmail.edu.au

Huaying Chen

CSIRO Manufacturing Flagship

Dan Yuan

University of Wollongong, dy983@uowmail.edu.au

Gursel Alici

University of Wollongong, gursel@uow.edu.au

See next page for additional authors

Follow this and additional works at: https://ro.uow.edu.au/eispapers

Part of the Engineering Commons, and the Science and Technology Studies Commons

Research Online is the open access institutional repository for the University of Wollongong. For further information contact the UOW Library: research-pubs@uow.edu.au 


\title{
Development of a novel magnetophoresis-assisted hydrophoresis microdevice for rapid particle ordering
}

\author{
Abstract \\ Focusing and ordering of micro- or nanoparticles is an essential ability in microfluidic platforms for bio- \\ sample processing. Hydrophoresis is an effective method utilising hydrodynamic force to focus \\ microparticles, but it is limited by the fixed operational range and the lack of flexibility. Here, we report a \\ work to tune and improve the dynamic range of hydrophoresis device using magnetophoresis. In this \\ work, a novel approach was presented to fabricate the lateral fluidic ports, which allow the flipped chip to \\ remain stable on the stage of microscope. Diamagnetic polystyrene microparticles suspended in a \\ ferrofluidic medium were repelled to the lower level of the channel by negative magnetophoretic force, \\ and then interact with grooves of microchannel to obtain an excellent hydrophoretic ordering. The effects \\ of (i) flow rate, (ii) particle size, (iii) magnetic susceptibility of the medium, and (iv) number of magnets on \\ the particle focusing efficiency were also reported. As the proposed magnetophorsis-assisted \\ hydrophoretic device is tuneable and simple, it holds great potential to be integrated with other \\ microfluidic components to form an integrated sample-to-answer system.

\section{Disciplines} \\ Engineering | Science and Technology Studies

\section{Publication Details} \\ Yan, S., Zhang, J., Chen, H., Yuan, D., Alici, G., Du, H., Zhu, Y. \& Li, W. (2016). Development of a novel \\ magnetophoresis-assisted hydrophoresis microdevice for rapid particle ordering. Biomedical \\ Microdevices, 18 (4), 54-1-54-9.

\section{Authors} \\ Sheng Yan, Jun Zhang, Huaying Chen, Dan Yuan, Gursel Alici, Haiping Du, Yonggang Zhu, and Weihua Li
}




\title{
Development of a novel magnetophoresis-assisted hydrophoresis microdevice for rapid particle ordering
}

\author{
Sheng Yan ${ }^{1}$ Jun Zhang ${ }^{1}$ Huaying Chen ${ }^{2}$ Dan Yuan ${ }^{1}$ Gursel Alici ${ }^{1,3}$ Haiping Du ${ }^{4}$ \\ Yonggang $\mathrm{Zhu}^{2, \mathrm{a}}$ and Weihua $\mathrm{Li}^{1, \mathrm{a}}$ \\ ${ }^{1}$ School of Mechanical, Materials and Mechatronic Engineering, University of Wollongong, Wollongong, NSW 2522, \\ Australia. \\ ${ }^{2}$ CSIRO Manufacturing Flagship, Private Bag 10, Clayton South, VIC, 3169, Australia. \\ ${ }^{3}$ ARC Centre of Excellence for Electromaterials Science, Innovation Campus, University of Wollongong, Wollongong, \\ NSW 2500, Australia \\ ${ }^{4}$ School of Electric, Computer and Telecommunication Engineering, University of Wollongong, Wollongong, NSW \\ 2522, Australia
}

\begin{abstract}
Focusing and ordering of micro- or nanoparticles is an essential ability in microfluidic platforms for bio-sample processing. Hydrophoresis is an effective method utilising hydrodynamic force to focus microparticles, but it is limited by the fixed operational range and the lack of flexibility. Here, we report a work to tune and improve the dynamic range of hydrophoresis device using magnetophoresis. In this work, a novel approach was presented to fabricate the lateral fluidic ports, which allow the flipped chip to remain stable on the stage of microscope. Diamagnetic polystyrene microparticles suspended in a ferrofluidic medium were repelled to the lower level of the channel by negative magnetophoretic force, and then interact with grooves of microchannel to obtain an excellent hydrophoretic ordering. The effects of (i) flow rate, (ii) particle size, (iii) magnetic susceptibility of the medium, and (iv) number of magnets on the particle focusing efficiency were also reported. As the proposed magnetophorsis-assisted hydrophoretic device is tuneable and simple, it holds great potential to be integrated with other microfluidic components to form an integrated sample-to-answer system.
\end{abstract}

Keywords Magnetophoresis hydrophoresis particle ordering tunable

\footnotetext{
a) Author to whom correspondence should be addressed. Electronic mail: weihuali@uow.edu.au and yonggang.zhu@csiro.au
} 


\section{Introduction}

In the last few decades, there is a trend towards miniaturization and integration of chemical or biological laboratories into a microfluidic chip, which has several promising advantages, such as low costs, portability, precise manipulation of fluid and particles, lower volume reagents and rapid analysis. It is a routine procedure to accurately handle the specific micro- or nanoparticles conjugated with biological or chemical species of interest for characterization and measurement purposes (Kawaguchi 2000, Tan et al. 2007). Particles or cells are typically focused into an identical streamline by flow cytometry for a more accurate analysis (Crosland-Taylor 1953). The problem with this approach is that it is bulky, expensive and requires sheath flows to focus particles. It is crucial to develop novel approaches enabling the devices cheap, accessible and portable.

Groove-based microfluidic focusing is one such method. Ligler's group (áHowell Jr 2008, Golden et al. 2009 , Kim et al. 2009, Thangawng et al. 2010) proposed microflow cytometers using groove-generated sheath flow for cell assay and bacteria detection. However, the focusing performance highly depends on the accurate control of flow rate. Hydrophoresis is a sheathless method, an emerging passive approach that utilizes the steric effects between particles and grooves (Choi et al. 2008). The hydrophoretic devices have so far been demonstrated for many biological applications, i.e. blood cell separation (Choi et al. 2007, Choi et al. 2011), cell cycle synchronization (Choi et al. 2009), focusing of mammalian cell lines (Choi et al. 2008) and cell removal (Song et al. 2014). However, the hydrophoresis method is limited by its fixed operation range. The devices are designed and fabricated with appropriate dimensions for specific assays. Once the target particles are changed to new sets, the hydrophoretic channel has to be redesigned and refabricated to satisfy the new requirements. Choi and Park (Choi et al. 2009) previously reported a tunable hydrophoretic device fabricated in poly-(dimethylsiloxane) (PDMS) where the channel height was reduced by applying compressive stress to the device. Another approach to tune hydrophoresis was implemented by our group (Yan et al. 2014, Yan et al. 2014, Sheng et al. 2015, Yan et al. 2015) using a dielectrophoresis (DEP)-assisted hydrophortic device where interdigitated electrodes were embedded on the bottom of the channel, generating negative DEP to assist particles in forming hydrophoretic ordering. Alternatively, replacing micro-electrodes with a diaphragm could tune the critical diameter of hydrophoretic devices via the applied pressure on the diaphragm (Yan et al. 2014). Tunable devices are demanding in a live feedback system, where the parameters of microchannel can be adjusted on real-time to retrieve the expected results without fabricating new devices.

Magnetophoresis, a motion of particles under the effects of magnetic fields, is a simple manipulation technique that has gained considerable attention in the last decade. Due to its non-contact nature and independent of solution $\mathrm{pH}$, ionic concentration, surface charge and temperature, a number of techniques using megnetophoresis have been reported in many biological studies (Shen et al. 2012). To manipulate cells that are non-magnetic in nature, functionalized magnetic beads were 
conjugated with cells of interest through either enbocytosis or ligand-receptor interaction at their surface (Robert et al. 2011). Plouffe et al. (Plouffe et al. 2011) presented an optimised magnetophoretic device for isolating a magnetic-particle-tagged cell population from a homogeneous suspension. Forbes et al. (Forbes et al. 2012) introduced a magnetophoretic separation of immunomagnetically labelled rare mammalian cells using an angled magnet. As positive magnetophoresis involves in cellbead conjugation for specific manipulation, the whole assay lasts longer due to several hours' incubation. Besides, multiple washing steps are labour-intensive and may cause cell loss (Pamme et al. 2006, Robert et al. 2011). In positive magnetophoresis, microparticles will be attracted towards the maximum magnetic strength, however, in negative magnetophoresis, they will be repelled to the opposite direction: the minimum magnetic strength. Negative magnetophoresis is a label-free manipulative technique, which can address the above-mentioned issues (Kose et al. 2009). In this case, the diamagnetic particles or cells are dispersed in a magnetic fluid such as a paramagnetic salt medium (Rodriguez-Villarreal et al. 2011, Shen et al. 2012)or a ferrofluid (Zhu et al. 2011, Zhu et al. 2014). In magnetophoretic devices, magnetic forces should overcome drag forces to precisely control the particle movements. Particles of interest laterally migrate at a larger distance to distinguish from their mixtures (Hejazian et al. 2015).

In this work, we propose a magnetophoresis-assisted hydrophoretice device, which takes the advantages of the both methods. Unlike other magnetophoresis-based techniques that drive the particles to move a long distance in the lateral direction, the proposed method utilizes megnetophoretic force to push particles downwards in the height direction, which then couples with hydrophoretic effect to form particle ordering. Magnetophoresis as an active method can relax the restriction of height dimension of hydrophoretic devices. The outline of this paper is as follows: Materials and methods section will introduce the methodology, fabrication, material preparation, experimental setup and numerical simulation. The main results will be presented in Results and discussion, including the effects of flow rate, particle diameter, ferrofluidic concentration and number of magnets on the focusing efficiency. The main conclusion will be drawn in Conclusion section.

\section{Materials and methods}

\subsection{Methodology}

Hydrophoresis employs anisotropic grooves to generate helical flow patterns in the microfluidic channel. Typically, particles travel back and forth along the transverse direction following the vortexes (Fig. 1a). However, particle-groove interaction plays a dominant role once the diameter of the particles exceeds the half of the channel height. The particle-groove interaction diffuses the particles out of their streamlines and leads to a new equilibrium position; this is called hydrophoretic ordering and the critical diameter to be focused in the hydrophoretic channel is defined as the half of the channel height (Yan et al. 2014). 
To overcome the lack of flexibility in focusing small particle whose diameter is less than the critical diameter, megnetophoresis is involved to extend the operational range of hydrophoretic device. The magnetic force $\left(F_{\text {mag }}\right)$ exerting on a particle (Eq. (1)) relies on the difference between the magnetic susceptibility of the particle $\chi_{p}$ and the base fluid $\chi_{m}$, as well as the volume of the particle, $V_{p}$, the magnetic flux density and gradient of the magnetic field $(\mathbf{B} \cdot \nabla) \mathbf{B}$, and $\mu_{0}$, the permeability of free space $\left(4 \pi \times 10^{-7} \mathrm{H} \mathrm{m}^{-1}\right)$ (Peyman et al. 2009):

$$
\mathbf{F}_{\text {mag }}=\frac{\left(\chi_{p}-\chi_{m}\right) V_{p}}{\mu_{0}}(\mathbf{B} \cdot \nabla) \mathbf{B}
$$

For a magnetic particle $\left(\chi_{p}>0\right)$ dispersed in an aqueous diamagnetic medium $\left(\chi_{m}<0\right)$, a positive magnetophoresis is generated in this mode, where the particle migrates to the region of a strong magnetic field. On the contrary, if the particle is diamagnetic $\left(\chi_{p}<0\right)$ and the medium is paramagnetic $\left(\chi_{m}>0\right)$ then the difference between the values becomes negative and the particle is repelled from the magnetic field towards an area of field minima.

In our experiments, the diamagnetic particles were experienced two significant forces: magnetic repulsion force and drag force (Fig. S1 in the Supporting Information). The drag force in the $z$-axis is expressed as: $F_{d z}=-6 \pi R \eta\left(v_{m z}-v_{p z}\right)$, where R is the radius of the particle, $\eta$ is the viscosity of the medium, $v_{m z}$ and $v_{p z}$ are the $z$-velocity of the flow and the particle (Massey 1989). Typically, magnetic repulsion force should be larger than the z-component of the drag force, which can be expressed as: $\left|F_{d z}\right|<\left|F_{\text {mag }}\right|$. Therefore, a dimensionless parameter in our magnetophoresis-assisted hydrophoretic system can be defined as:

$$
1<\frac{\left|F_{m a g}\right|}{\left|F_{d z}\right|}=\frac{2}{9 \mu_{0} \eta} \cdot \frac{\left|\chi_{p}-\chi_{m}\right|}{\left|v_{m z}-v_{p z}\right|} \cdot(\mathbf{B} \cdot \nabla) \mathbf{B} \cdot R^{2}
$$

This term encompasses all the relevant variables into a single dimensionless parameter. At a lower flow rate, the magnetic force can easily overcome the drag force and push particles downwards to the lower level of the channel, where the intensive particle-groove interactions enable the particles to form hydrophoretic ordering (Fig. 1b). Also, the particle with a larger radius can be easily focused. Besides, increasing the magnetic field and susceptibility mismatch of the particle and the fluid would be alternatives in focusing particles.

\subsection{Fabrication}

Fig 1c shows the optical images of hydrophoretic channel. The hydrophoretic channel consisted of a series of grooves with a small curvature of $600 \mu \mathrm{m}$ and a large curvature of $650 \mu \mathrm{m}$. Both channel height and groove height were $40 \mu \mathrm{m}$. The channel was $600 \mu \mathrm{m}$ wide and $20 \mathrm{~mm}$ long.

Fig. 2a shows a schematic diagram of the overall procedures for fabrication of the microfluidic platform with L-shaped interconnections. Sylgard 184 elastomer base and curing agent (Dow Corning Corporation, Midland, USA) were completely 
mixed at a weight ratio of 10:1 and degassed under vacuum. Subsequently, a small portion of the PDMS mixture was cast against the mold that was fabricated by standard photolithography ( $\mathrm{Li}$ et al. 2012, Yan et al. 2015). The thickness of the first PDMS casting was remained to be approximately $1 \mathrm{~mm}$ (Fig. 2a I). Such thin layer was convenient for removing the residual PDMS fragments after punching the vertical holes. Afterwards, the PDMS mixture on the mold was baked in an oven at 65 ${ }^{\circ} \mathrm{C}$ for $45 \mathrm{~min}$ and then taken out from the oven before complete curing. The modified syringe needles were placed on the corresponding inlet and outlet. To obtain the modification of syringe needles, syringe needles ( $25 \mathrm{G}$, Terumo needle, Terumo Corporation) were sawed at the neck in order to get rid of the plastic inlet (Fig. S2 in the Supporting Information). The sticky surface of PDMS can prevent the modified needles from rolling when implementing second PDMS casting. To avoid the penetration of PDMS by the cutting tips (Harris Uni-Core Punch; Ted Pella, Redding, CA, USA), the second PDMS layer was controlled to be around $3 \mathrm{~mm}$ in depth (Fig. 2a II). The step (II) can be repeated multiple times to fabricate the three dimension (3D) microfluidic ports. The cured PDMS replica was peeled off from the mold, with pulling off the needles from PDMS using a pair of tweezers. The vertical holes for inlet and outlet ports were punched to the lateral holes, rather than cutting through the whole PDMS slab, using a cutting tip $(\varnothing 0.75 \mathrm{~mm})$. The residual PDMS fragments produced by the cutting tips were carefully removed by tweezers (Fig. 2a III). Both the PDMS replica and a glass slide were then treated using a plasma cleaner (PDc-002, Harrick Plasma, Ossining, NY) for $3 \mathrm{~min}$, before they were brought into conformal contact (Fig. 2a IV). The L-shaped interconnections were then connected to Tygon tubing ( $0.7 \mathrm{~mm}$, Tygon R-AAQ04103; Saint-Gobain Performance Plastics, Akron, OH, USA) using 21-gauge flat-ended needles.

Conventionally in a through-hole type interconnection for particle injection, the flat-ended needles are used to penetrate the chip and connected through inlet and outlet holes that are vertical to the flow direction (Fig. 2b). Once the chip is flipped, the flat-ended needles with Tygon tubing are unable to keep the chip stable on the travelling stage of microscope. Furthermore, the flat-ended needles increase the distance between micro-chip and objective lens, which may cause the captured images out of focus. In the current study, the L-shaped interconnection is proposed as a remedy for the above issues (Fig. 2c). The micro-chip can be easily flipped without any unstable and out-of-focus issues. A schematic drawing of the whole device is shown in Fig. 2d.

\subsection{Material preparation}

A commercial water-based magnetite ferrofluid (EMG 408, Ferrotec Co., NH) was used in our experiments. The volume ratio of the magnetite particles for this ferrofluid is $1.1 \%$. The mean diameter of nanoparticles is $10.2 \mathrm{~nm}$. The initial magnetic susceptibility is measured to be 0.26 ; the saturation magnetization $\left(\mu_{0} \mathrm{M}\right)$ is 60 Gauss; the dynamic viscosity is $1.2 \times$ $10^{-3} \mathrm{~kg}\left(\mathrm{~m} \mathrm{~s}^{-1}\right.$ (Zhu et al. 2011). The fluorescent microparticles with diameters of 5, 8, 10, $13 \mu \mathrm{m}$ (Coefficient of variation 
$5 \%, 18 \%, 5 \%, 16 \%)$ were purchased from Thermo Fisher Scientific Corporation. The microbeads were re-suspended in $\times 0.02$ EMG 408 ferrofluid to a final concentration of $10^{6}$ particles per millilitre. To optimise the focusing performance of the $5 \mu \mathrm{m}$ beads, the beads were re-suspended in $\times 0.05$ and $\times 0.1$ ferrofluids. To prevent the beads from sedimentation and aggregation, $0.1 \%$ (in volume) Tween 20 (Sigma-Aldrich, product no. P9416) surfactant was added to this aqueous medium.

Prior to each experiment, the device was rinsed with DI water at $20 \mu 1 \mathrm{~min}^{-1}$ for $20 \mathrm{~min}$ using a syringe pump. To prevent immediate particle adhesion to the PDMS surface, $0.1 \%$ Tween 20 in DI water was injected into the microchannel and incubated at $37^{\circ} \mathrm{C}$ for 30 min to coat the PDMS surface.

\subsection{Experimental setup}

A non-uniform magnetic field was generated by a stack of three NdFeB permanent magnets. Each magnet is 2 mm in width, $2 \mathrm{~mm}$ in length, and $2 \mathrm{~mm}$ in thickness. The magnets stack was placed on the top of glass as indicated in Fig. 2 d. The magnetic flux density at the center of the magnets' pole surface was measured to be $300 \mathrm{mT}$ by a Gauss meter (Model 5180 , Pacific Scientific OECO). The magnetization direction of these magnets is perpendicular to the hydrophoretic channel. A syringe pump (Legato $100, \mathrm{Kd}$ Scientific) was used to inject the particle suspension into the micro-channel. All images were captured through an inverted microscope (CKX41, Olympus, Japan) with a CCD camera (Rolera Bolt, Q-imaging, Australia) and an image processing program (Q-Capture Pro 7, Q-imaging, Australia).

\subsection{Numerical simulation}

The particle trajectories in microchannel were numerically modelled using finite element software (COMSOL Multi-physics 5.0 COMSOL, Burlington, MA). The 3D modelling for simulation was identical to the experimental channel. With magnetic fields, the particle trajectories were simulated by coupling Laminar Flow module, Magnetic Fields module and Particle Tracing module. First, the Laminar Flow module was utilised to investigate the fluid flow inside the 3D channel. No-slip boundary conditions were set to the channel walls. The flow was assumed to be incompressible. The flow rate released at the inlet was $20 \mu \mathrm{min}^{-1}$ and the pressure at the outlet was zero. The Magnetic Fields module was employed to calculate the nonuniform magnetic fields generated by the permanent magnets. A Faraday cage was also built to contain the hydrophoretic channel and magnet to exclude electrostatic and electromagnetic influences (Fig. S3a in the Supporting Information). The Zero Magnetic Scalar Potential was chosen from one of the Faraday cage's corners. A stationary solver was used to compute the magnetic field (Fig. S3b in the Supporting Information). Finally, the Particle Tracing module was used to couple the magnetophoretic force and the hydrodynamic drag force to predict the particle trajectories. The equations of the magnetophoretic force and drag force embedded in the COMSOL software were used without modifications. At the inlet, particles were evenly released along the channel width. The particle density and diameter were set to $1050 \mathrm{~kg} \mathrm{~m}^{-3}$ and $13 \mu \mathrm{m}$, 
respectively. The time for beads migrating in the channel was set to $5 \mathrm{~s}$ and the time interval for each step was set to $0.001 \mathrm{~s}$, which provided smooth particle trajectories. The Free Tetrahedral was used in the model mesh.

\section{Results and discussion}

\subsection{Magnetophoresis-assisted hydrophoretic ordering}

First, the effect of negative magnetophoresis to assist the hydrophoretic ordering was investigated. Using the device with hydrophoretic channel, ferrofluid and magnets, the different patterns were observed when beads passed through the channel. Figs. 3a and $\mathrm{b}$ show the simulated and experimental particle trajectories in the magnetophoresis- assisted hydrophoretic device without a magnetic field. The flow rate applied in the numerical model and experiment was $20 \mu 1 \min ^{-1}$ and the beads injected into the channel were $13 \mu \mathrm{m}$. Since the particle diameters were smaller than half of the channel height, they could not meet the requirement of hydrophoretic ordering and thus migrated back and forth inside the channel. After applying the magnetic field, the particles released evenly from the inlet were pushed by the magnetic repulsion force towards to the lower level of the channel, where the particle-groove interactions diffused particles out of helical motion and drove particles to a new equilibrium position (Fig. 3c). Fig. 3d shows the optical micrographs of particle trajectories of $13 \mu \mathrm{m}$ beads passing through the magnetophoreisis-assisted hydrophoretic device. The measured trajectories agree reasonably well with the simulation result shown in Fig. 3c. Regardless of the initial positions, all the beads were focused onto the sidewalls of the channel.

\subsection{Effect of flow rate}

The dependence of magnetophoretic-assisted hydrophoretic particle ordering on the flow rate was verified as the flow rate ranged from 10 to $80 \mu \mathrm{min}^{-1}$ in the presence of magnetic field. The particles with a diameter of $13 \mu \mathrm{m}$ were used as a model. When introduced into the channel, the particles will experience both the inertial and viscous forces. To examine the relative effect of these two forces, we calculated the particle Reynolds number $\mathrm{R}_{\mathrm{p}}$ which is the dimensionless ratio of particle inertia to the viscous force (Choi et al. 2009), i.e. $\mathrm{R}_{\mathrm{p}}=\left(\rho d^{2} u\right) /\left(\mu D_{h}\right)$, where $D_{h}$ is the hydraulic diameter defined as $D_{h}=2 w h /(w+h)$. For $\mathrm{R}_{\mathrm{p}}>1$, the particle inertia plays a dominant role and the equivalent paths of particles under hydrophoretic ordering are also affected by the inertial force. To simplify the calculation, the presence of the grooves was ignored, so the linear velocities of particles passing through the $600 \mu \mathrm{m}$ wide by $40 \mu \mathrm{m}$ high channel ranged from 0.69 to $5.53 \mathrm{~cm} \mathrm{~s}^{-1}$. The resulting particle Reynolds number is 0.12 for $13 \mu \mathrm{m}$ beads at the maximum working flow rate of $80 \mu 1 \mathrm{~min}^{-1}$. Therefore, under the present experimental conditions, the particle inertial effect can be omitted.

Fig. 4a shows the focusing efficiency of $13 \mu \mathrm{m}$ particles under various flow rates. The focusing efficiency is defined as $\mathrm{E}_{\mathrm{n}}=(w-d / 2-a) /(w-d)\left(\right.$ Choi et al. 2008), where $a$ represents the width of the focused particle stream, $a=a_{1}+a_{2}$ (Fig. 4b). Below 
$20 \mu \mathrm{min}^{-1}$, the flow rate had little effect on the focusing efficiency because the magnetic repulsion force played a dominant role and the particles travelled in a limited space where the particle-groove interactions were enough to focus them well (Fig. $4 \mathrm{~b}$ and $\mathrm{c})$. In contrast, the exposure time to magnetic repulsion force was not long enough to keep particles focused well when the particles passed through the channel at a higher flow rate. The focusing efficiency decreased from $82.5 \pm 2.2 \%$ to $76.4 \pm 2.0 \%$ with the increase of flow rate from 30 to $50 \mu 1 \mathrm{~min}^{-1}$ (Fig. $4 \mathrm{~d}$ and e). Above $80 \mu 1 \mathrm{~min}^{-1}$, the particles occupied the entire channel at the outlet, which meant they could not form self-ordering any longer (Fig. 4f).

\subsection{Effect of particle diameter}

As indicated in Eq. (2), the volume of microsphere is an important parameter to determine the magnetic force. To investigate the effect of diameter on the focusing efficiency, $5,8,10$, and $13 \mu \mathrm{m}$ polystyrene particles were suspended in $\times 0.02 \mathrm{EMG}$ 408 ferrofluid and pumped through the microchannel. They were individually introduced into the microfluidic channel at a

flow rate of $20 \mu \mathrm{L} \mathrm{min}{ }^{-1}$. Before encountering the magnetic field created by magnets, the particles were evenly distributed across the width of the channel. When passing through the magnetic field, the particles experiencing repulsion forces were pushed downwards in the microchannel.

Figs. 5a-c shows the focusing profiles of different particle diameters at the outlet without a magnetic field (Left) and with a magnetic field (Right). Since the larger particles (i.e, 10 and $13 \mu \mathrm{m}$ beads) exerting larger magnetic repulsion force could easily form hydrophoretic ordering, narrower focusing bands were observed. The focusing efficiency of 10 and $13 \mu \mathrm{m}$ beads reached to $90 \pm 2 \%$ and $89 \pm 2.5 \%$, respectively (Fig. 5 d). Based on the experimental results, the focusing efficiency of $13 \mu \mathrm{m}$ beads was slightly lower than that of $10 \mu \mathrm{m}$ beads due to the wider variation of $13 \mu \mathrm{m}$ particles $(\mathrm{CV}: \sim 16 \%)$. In contrast, the focusing efficiency dropped to $64.1 \pm 3 \%$ for $8 \mu \mathrm{m}$ beads, and $40.4 \pm 2.2 \%$ for $5 \mu \mathrm{m}$ beads (Fig. $5 \mathrm{~d}$ ). Even though $5 \mu \mathrm{m}$ particles had a tendency to focus onto the sidewalls of the channel, the low focusing efficiency proved that helical flows were dominant in the particle motion. However, increasing the concentration of ferrofluid and number of magnets would be good options to improve the focusing efficiency of $5 \mu \mathrm{m}$ beads.

\subsection{Effect of ferrofluidic concentration and number of magnets}

As demonstrated above, small particles were not focused well in the $\times 0.02$ ferrofluid. To demonstrate the tunability of magnetophoretic-assisted hydrophoretic device, more magnets and higher concentration of ferrofluid were used to improve the focusing efficiency of $5 \mu \mathrm{m}$ beads. The concentration of ferrofluid can affect the magnetic susceptibility of the medium $\left(\chi_{m}\right)$. Eq. (2) illustrates that increasing $\chi_{m}$ results in increased relative susceptibility between particles and medium, and therefore greater magnetic repulsion force applies on the diamagnetic particles. Here, the ferrofluid was added to the particle 
suspending with a final dilution of 0.05 and 0.1 to investigate the effect of ferrofluidic concentration on focusing efficiency. In addition, more magnets arranged on the top of the channel can not only increase the exposure time to the magnetic field, enabling beads to migrate a larger distance, but also enhance the magnetic field, which in turn affects the magnetic force. The $5 \mu \mathrm{m}$ beads were injected through the channel with a flow rate of $20 \mu \mathrm{L} \mathrm{min}^{-1}$ with different numbers of magnets placed in a row as indicated in Fig. 2e.

Figs. $6 \mathrm{a}$ and $\mathrm{b}$ shows the focusing profiles of $5 \mu \mathrm{m}$ beads at the outlet with different working conditions. As the number of magnet increased from 2 to 4 in $\times 0.05$ ferrofluid, the beads were prone to focus along the sidewalls of channel with narrower bands. The focusing efficiency increased dramatically from $14.4 \%$ to $52.4 \%$ (Fig. 6c). The improvement was attributed to the longer exposure time under the magnetic field and stronger magnetic field. At $20 \mu \mathrm{L} \mathrm{min}^{-1}$, the average velocity of beads in the channel is $13.8 \mathrm{~mm} \mathrm{~s}^{-1}$. The particle residence time under the magnetic field was $0.29 \mathrm{~s}, 0.43 \mathrm{~s}$ and $0.58 \mathrm{~s}$, separately, with the number of magnet from 2 to 4 . The greater residence time in 4-magnet setup allowed particles to migrate a longer distance over a greater period. Besides, it should be noted that the intensity of magnetic field varied with the number of magnet. The values of magnetic field at a distance of $1 \mathrm{~mm}$ (the thickness of glass) were 119, 149 and $190 \mathrm{mT}$. Thus, as the particles passed through the magnetic field, they experienced a slightly larger repulsive effect in 4-magnet setup.

Although similar behavior was observed in $\times 0.1$ ferrofluid, the focusing efficiency was higher when compared to that in $\times 0.05$ ferrofluid. The $\times 0.1$ ferrofluid with more magnetic material content increased the magnetic susceptibility of the medium, resulting in larger magnetic force. The focusing efficiency increased from $52.4 \%$ to $62.0 \%$ in the presence of 4 magnetic blocks, which was the best result in the work. However, the focusing efficiency could be further improved by increasing ferrofluidic concentration and using a larger magnet with stronger magnetic field. Alternatively, particle trajectories can be altered by an electromagnet whose magnetic field can be tuned by the applied current.

\section{Conclussions}

The work proposed a new concept of magnetophoresis-assisted hydrophoretic ordering using a simple setup of inexpensive permanent magnets, and without the need for high-powered instruments (e.g. lasers or high voltage power supply). The critical limitation of magnetophoresis-based device operating at a low flow rate and the specific hydrophoretic device for focusing a given size of particles were overcome by this new microfluidic platform. To implement this method, a novel lateral fluidic port was introduced to keep the flipped chip stable. The motion of the microparticles was investigated and explained using numerically simulated particle trajectories and experimental results with and without the magnetic field. A high-throughput focusing was achieved with this new magnetophoresis-assisted hydrophoretic system, even when the flow 
rate was increased to $50 \mu \mathrm{L} \mathrm{min}$. The device has been demonstrated to successfully focus polystyrene fluorescent particles with $5,8,10$, and $13 \mu \mathrm{m}$ diameters. To achieve the better focusing efficiency of $5 \mu \mathrm{m}$ beads, the higher concentration of ferrofluid and more magnets were applied to tune the focusing patterns. This novel approach represents a versatile tool for rapid, label-free focusing. It can also facilitate the connection with other microfluidic platforms for downstream sorting and /or analysis.

\section{Acknowledgements}

This work is supported by the University of Wollongong -China Scholarship Council joint scholarships and the CSIRO Office of Chief Executive (OCE) Top-up Scholarship.

\section{References}

P. B. áHowell Jr. Two simple and rugged designs for creating microfluidic sheath flow. Lab Chip 8(7): 1097-1103 (2008).

S. Choi, et al., Hydrophoretic high-throughput selection of platelets in physiological shear-stress range. Lab Chip 11(3): $413-418$ (2011).

S. Choi, et al., Tuneable hydrophoretic separation using elastic deformation of poly(dimethylsiloxane). Lab Chip 9(13): 1962-1965 (2009).

S. Choi, et al., Sheathless hydrophoretic particle focusing in a microchannel with exponentially increasing obstacle arrays. Anal. Chem. 80(8): 3035-3039 (2008).

S. Choi, et al., Continuous blood cell separation by hydrophoretic filtration. Lab Chip 7(11): 1532-1538 (2007).

S. Choi, et al., Hydrophoretic Sorting of Micrometer and Submicrometer Particles Using Anisotropic Microfluidic Obstacles. Anal. Chem. 81(1): 50-55 (2009).

S. Choi, et al., Microfluidic Self-Sorting of Mammalian Cells to Achieve Cell Cycle Synchrony by Hydrophoresis. Anal. Chem. 81(5): 1964-1968 (2009).

S. Choi, et al., Sheathless focusing of microbeads and blood cells based on hydrophoresis. Small 4(5): 634-641 (2008).

P. J. Crosland-Taylor. A Device for Counting Small Particles suspended in a Fluid through a Tube. Nature 171(4340): 37-38 (1953).

T. P. Forbes, et al., Microfluidic magnetophoretic separations of immunomagnetically labeled rare mammalian cells. Lab Chip 12(8): 1471-1479 (2012).

J. P. Golden, et al., Multi-wavelength microflow cytometer using groove-generated sheath flow. Lab Chip 9(13): 1942-1950 (2009).

M. Hejazian, et al., Lab on a chip for continuous-flow magnetic cell separation. Lab Chip 15(4): 959-970 (2015).

H. Kawaguchi. Functional polymer microspheres. Prog. Polym. Sci. 25(8): 1171-1210 (2000).

J. S. Kim, et al., Multiplexed detection of bacteria and toxins using a microflow cytometer. Anal. Chem. 81(13): 5426-5432 (2009).

A. R. Kose, et al., Label-free cellular manipulation and sorting via biocompatible ferrofluids. Proc. Natl. Acad. Sci. U.S.A. 106(51): 21478-21483 (2009).

M. Li, et al., Continuous particle focusing in a waved microchannel using negative dc dielectrophoresis. J. Micromech.Microeng. 22(9): 095001 (2012).

B. Massey. Mechanics of Fluids Chapman Hall. London, UK (1989).

N. Pamme, et al., Continuous sorting of magnetic cells via on-chip free-flow magnetophoresis. Lab Chip 6(8): 974-980 (2006).

S. A. Peyman, et al., Diamagnetic repulsion-A versatile tool for label-free particle handling in microfluidic devices. J. Chromatogr. A 1216(52): $9055-9062$ (2009).

B. D. Plouffe, et al., Computational design optimization for microfluidic magnetophoresis. Biomicrofluid. 5(1): 013413 (2011).

D. Robert, et al., Cell sorting by endocytotic capacity in a microfluidic magnetophoresis device. Lab Chip 11(11): 1902-1910 (2011).

A. I. Rodriguez-Villarreal, et al., Flow focussing of particles and cells based on their intrinsic properties using a simple diamagnetic repulsion setup. Lab Chip 11(7): 1240-1248 (2011).

F. Shen, et al., Label-Free Cell Separation Using a Tunable Magnetophoretic Repulsion Force. Anal. Chem. 84(7): 3075-3081 (2012).

Y. Sheng, et al., An integrated dielectrophoresis-active hydrophoretic microchip for continuous particle filtration and separation. J. Micromech.Microeng. 25(8): 084010 (2015).

S. Song, et al., Smart Microfluidic Pipette Tip Enabled by Flow-Rate Insensitive Particle Ordering. Small 10(20): 4123-4129 (2014).

W.-H. Tan, et al., A trap-and-release integrated microfluidic system for dynamic microarray applications. Proc. Natl. Acad. Sci. U.S.A. 104(4): 1146-1151 (2007).

A. L. Thangawng, et al., A hard microflow cytometer using groove-generated sheath flow for multiplexed bead and cell assays. Anal. Bioanal. Chem. 398(5): 1871-1881 (2010).

S. Yan, et al., Isolating plasma from blood using a dielectrophoresis-active hydrophoretic device. Lab Chip 14(16): 2993-3003 (2014).

S. Yan, et al., Making a hydrophoretic focuser tunable using a diaphragm. Biomicrofluid. 8(6): 064115 (2014).

S. Yan, et al., On-chip high-throughput manipulation of particles in a dielectrophoresis-active hydrophoretic focuser. Sci. Rep. 4: 5060 (2014).

S. Yan, et al., A hybrid dielectrophoretic and hydrophoretic microchip for particle sorting using integrated prefocusing and sorting steps. Electrophoresis 36(2): 284-291 (2015).

T. Zhu, et al., Combining positive and negative magnetophoreses to separate particles of different magnetic properties. Microfluid. Nanofluid. 17(6): 973982 (2014).

T. Zhu, et al., Focusing microparticles in a microfluidic channel with ferrofluids. Microfluid. Nanofluid. 11(6): 695-701 (2011). 


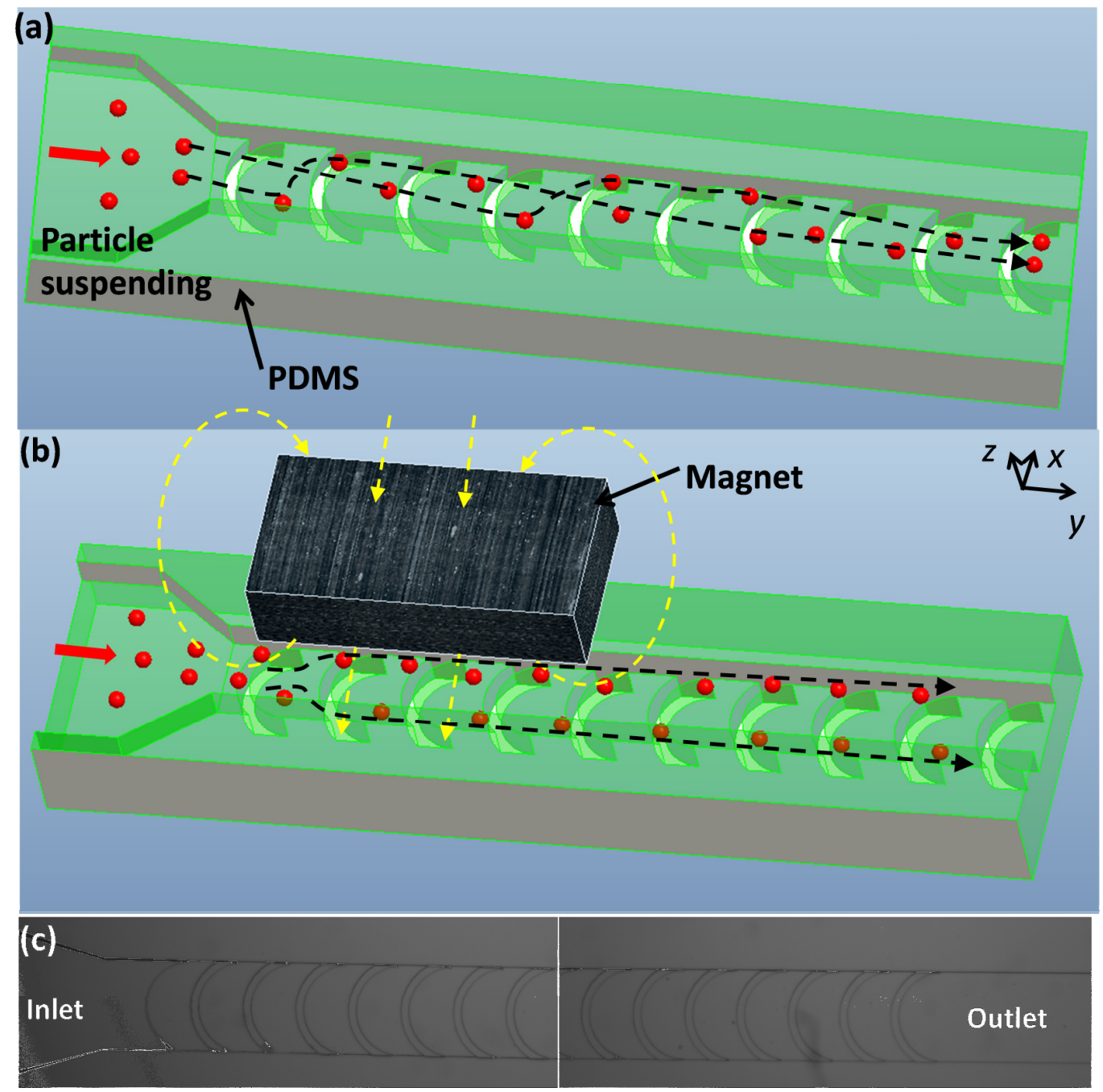

Fig. 1 Microfluidic device for hydrophoretic particle ordering. (a) Overview of the hydrophoretic device operation and structure. In the absence of magnetic field, randomly distributed particles migrate back and forth in the channel. (b) With the magnetic field, the particles were pushed downwards by the magnetic repulsion force, which enhances the particle-groove interactions. The particles satisfying the hydrophoretic ordering can be focused along the sidewalls of the channel. (c) Optical micrograph images of the hydrophoretic channel. 


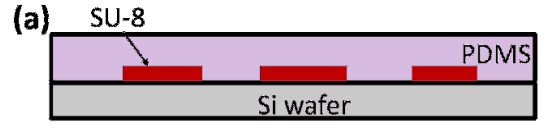

I. $1^{\text {st }}$ PDMS casting

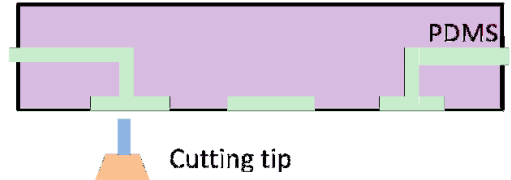

III. Removing the needles and punching the holes

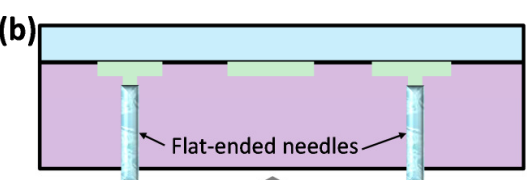

4X Objective len

Conventional fluidic ports

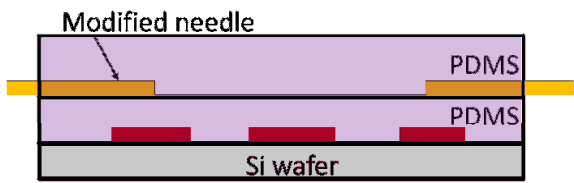

II. $2^{\text {nd }}$ PDMS casting

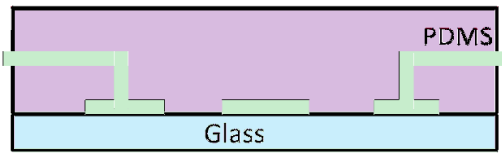

IV. Permanent bonding with glass slide

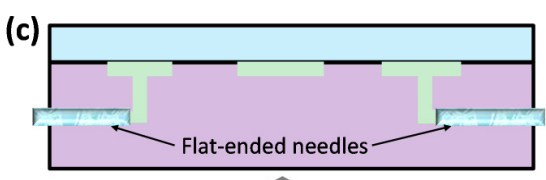

$4 X \quad$ Objective len

Lateral fluidic ports

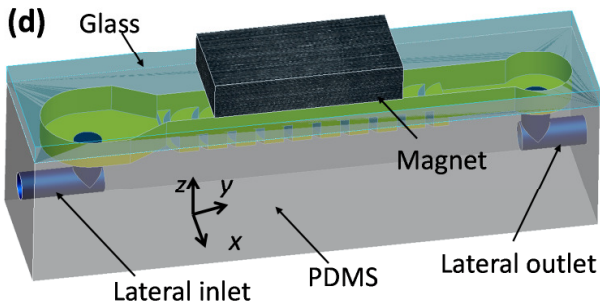

Fig. 2 (a) Schematic view of fabrication process flow. A schematic cross-sectional drawing of (b) the conventional fluidic ports and (c) the novel lateral fluidics ports. (d) Three dimensional animated model showing the overview of the device. 

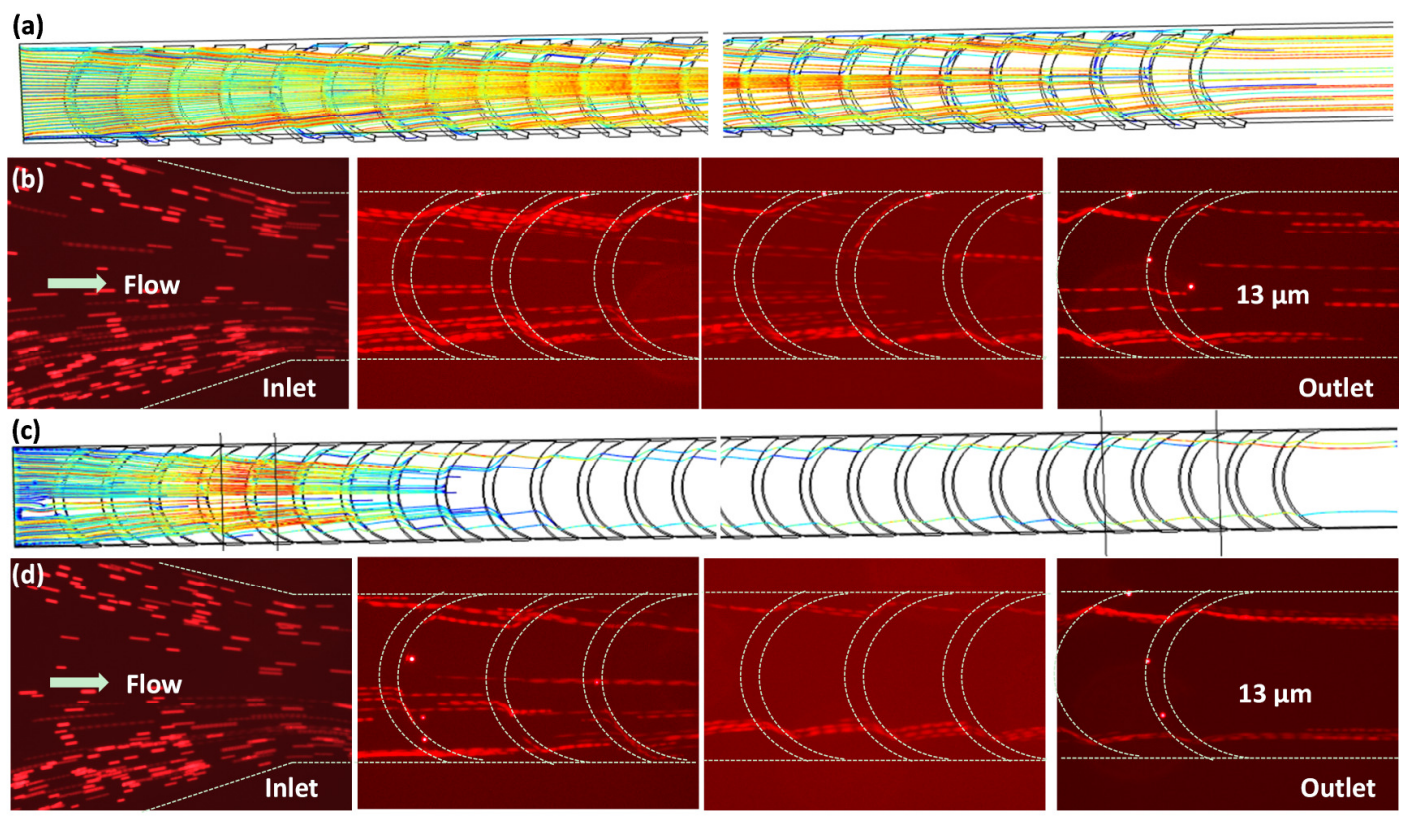

Fig. 3 Experimentally focused patterns and numerically predicted particle tracing of $13 \mu \mathrm{m}$ particles. The applied flow rate was $20 \mu 1$ min ${ }^{-1}$. The beads were evenly distributed at the inlet. (a) Simulated particle trajectories at both the inlet (Left) and the outlet (Right) without a magnetic field. Particles migrated back and forth were not focused in the channel. Red color represents high speed, blue low velocity. (d) Under a magnetic field, particles were focused onto the sidewalls of the channel once forming the hydrophoretic ordering. Optical microscopy images showing the focusing patterns (b) without a magnetic field and (d) with a magnetic field. 

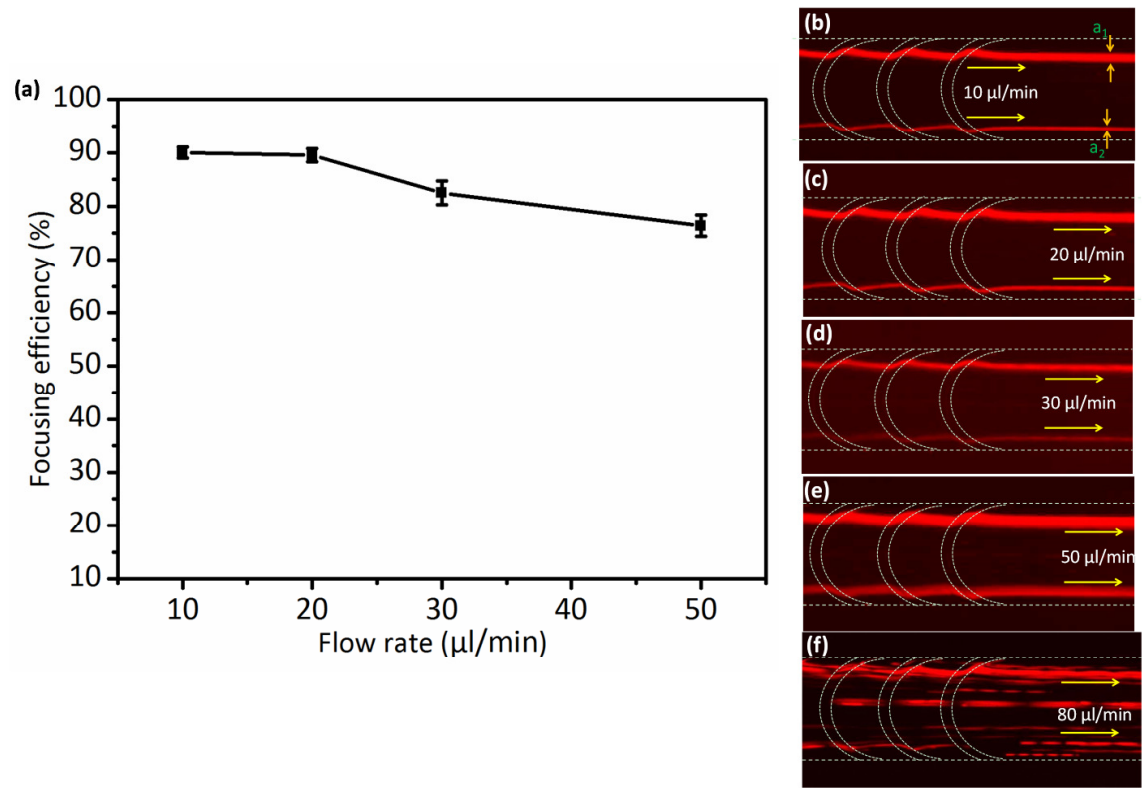

Fig. 4 (a) Measured focusing efficiency of $13 \mu \mathrm{m}$ particles. The flow rate ranged from 10 to $50 \mu 1 \mathrm{~min}^{-1}$ and a magnetic field was applied. The average value was 3 times the measurement. (b)-(f) Optical micrographs showing the particle trajectories in the channel at the flow rate of $10,20,30,50$ and $80 \mu l$ min ${ }^{-1}$. The average value was 3 times the measurement and the error bar represented the standard deviation. 

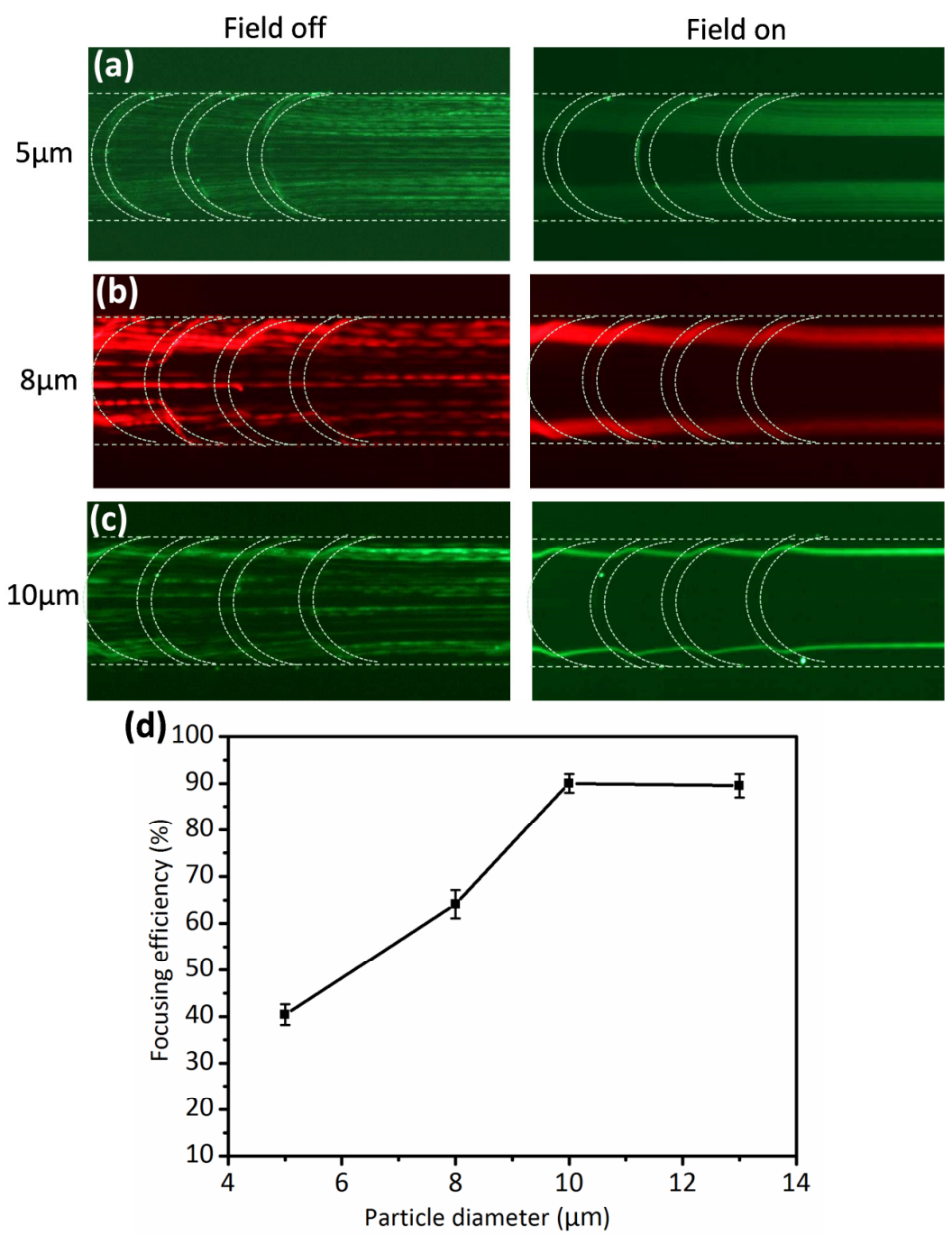

Fig. 5 (a)-(c) Optical micrographs showing the particle motion of 5, 8 and $10 \mu$ m beads at the outlet without a magnetic field (Left) and with a magnetic field (Right). The applied flow rate was $20 \mu \mathrm{min}^{-1}$. (d) Measured focusing efficiency of distinct particles. The average value was 3 times the measurement and the error bar represented the standard deviation. 

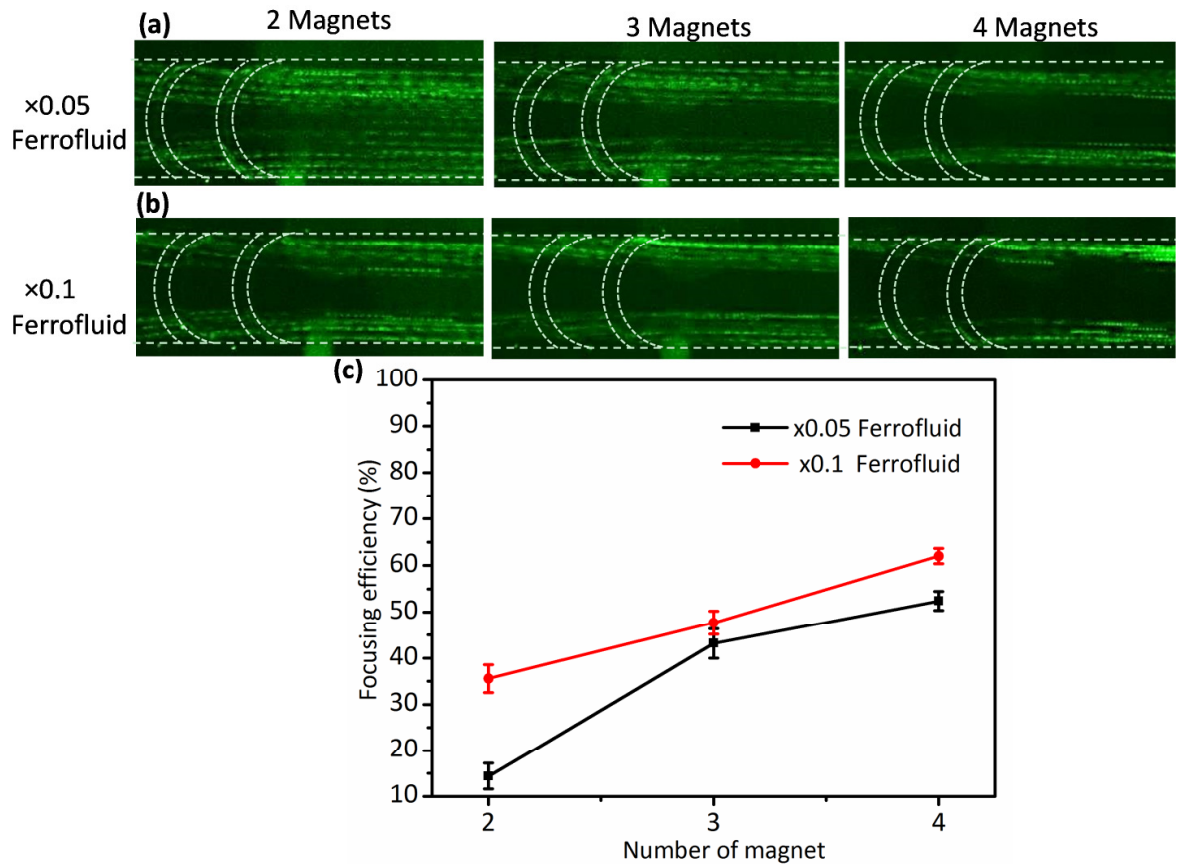

Fig. 6 Focusing patterns of $5 \mu \mathrm{m}$ beads at a flow rate of $20 \mu \mathrm{L} \mathrm{min}{ }^{-1}$ with different numbers of magnets, ranging from 2 to 4 . The optical microscopy images show the particle trajectories at the outlet in $\times 0.05$ ferrofluid (a) and $\times 0.1$ ferrofuid (b). (d) Measured focusing efficiency of $5 \mu$ m particles under different numbers of magnets and ferrofluidic concentration. The average value was 3 times the measurement and the error bar represented the standard deviation. 


\section{Supplementary material for}

\section{Development of a novel magnetophoresis-assisted hydrophoresis microdevice for rapid particle ordering}

Sheng Yan, Jun Zhang, Huaying Chen, Dan Yuan, Gursel Alici, and Haiping Du, Yonggang Zhu* and Weihua $\mathrm{Li}^{*}$

* To whom correspondence should be addressed. E-mail: weihuali@uow.edu.au; yonggang.zhu@csiro.au 


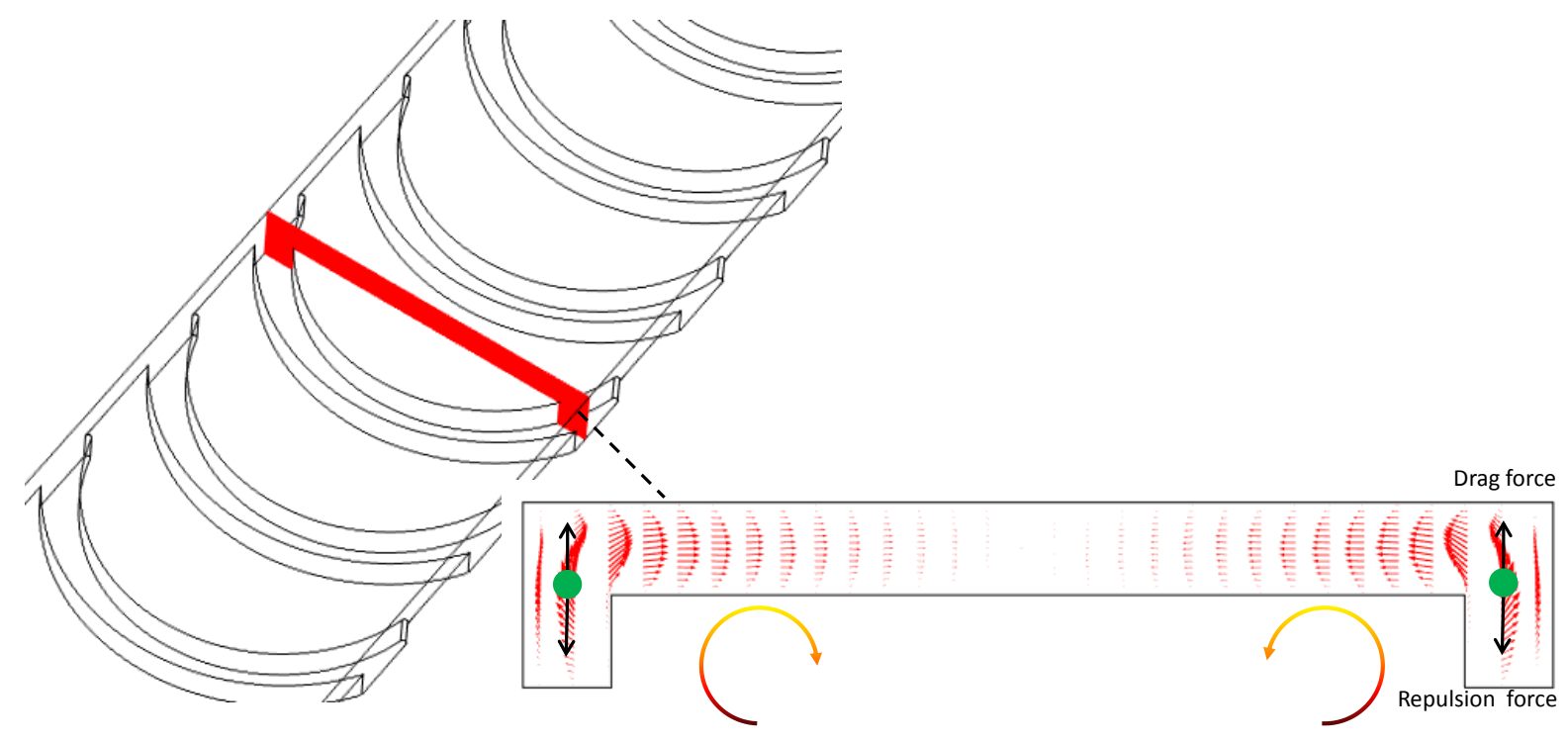

Figure S1. The schematic diagram of the micro-channel and the simulated results of flow field in the cross-sections. The anisotropic microstructure induces helical recirculations. The red arrows in the insets are velocity vectors.

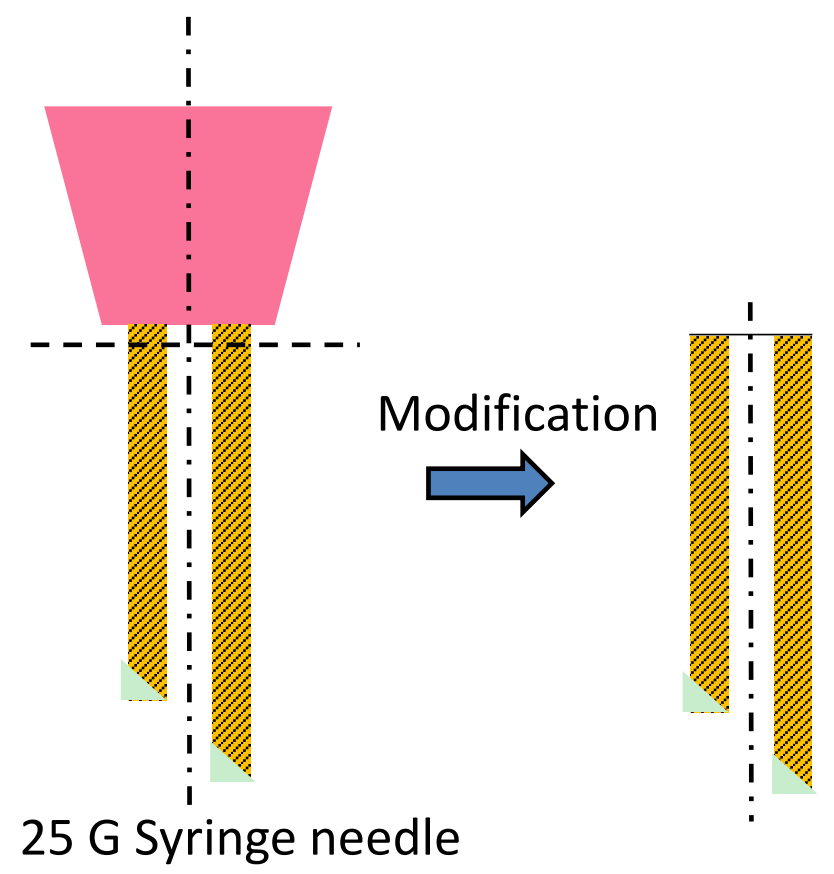

Figure S2. The modified needle was made by cutting the plastic part of $25 \mathrm{G}$ syringe needle. 
(a)

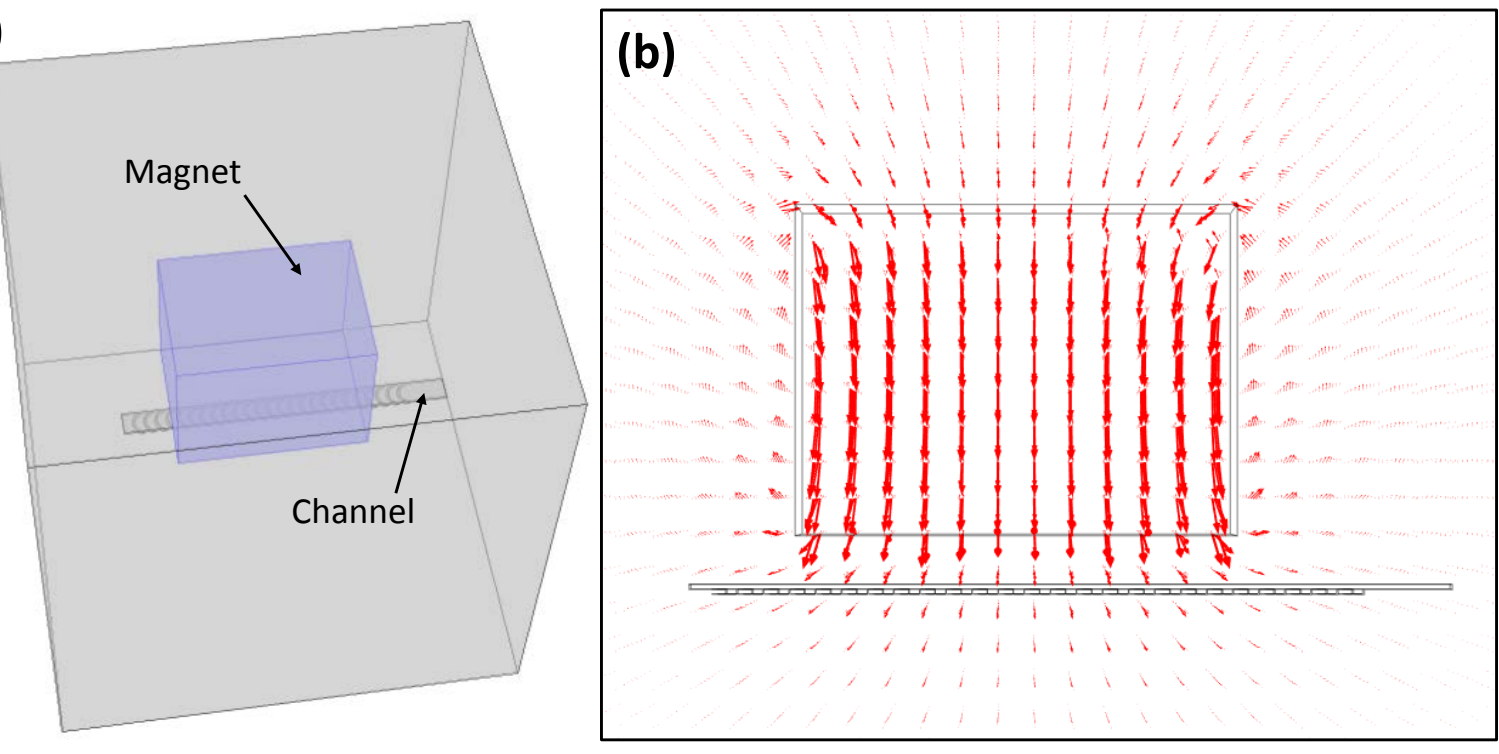

Figure S3. (a) The 3D full model considered; (b) The distribution of magnetic field generated by the magnet in the 3D model. The red arrows represent the vectors of magnetic field. 